\title{
TrES-4: A Transiting Hot Jupiter of Very Low Density
}

\section{Citation}

Mandushev, Georgi, Francis T. O'Donovan, David Charbonneau, Guillermo Torres, David W. Latham, Gáspár Á. Bakos, Edward W. Dunham, et al. 2007. “TrES-4: A Transiting Hot Jupiter of Very Low Density." The Astrophysical Journal 667 (2): L195-98. https://doi.org/10.1086/522115.

\section{Permanent link}

http://nrs.harvard.edu/urn-3:HUL.InstRepos:41397476

\section{Terms of Use}

This article was downloaded from Harvard University's DASH repository, and is made available under the terms and conditions applicable to Other Posted Material, as set forth at http:// nrs.harvard.edu/urn-3:HUL.InstRepos:dash.current.terms-of-use\#LAA

\section{Share Your Story}

The Harvard community has made this article openly available.

Please share how this access benefits you. Submit a story.

Accessibility 


\title{
TrES-4: A TRANSITING HOT JUPITER OF VERY LOW DENSITY
}

\author{
Georgi Mandushev, ${ }^{1}$ Francis T. O’Donovan, ${ }^{2}$ David Charbonneau, $, 4,4$ Gullermo Torres, ${ }^{3}$ David W. Latham, ${ }^{3}$ \\ Gáspár Á. Bakos, ${ }^{3,5}$ Edward W. Dunham, ${ }^{1}$ Alessandro Sozzetti, ${ }^{3,6}$ José M. Fernández, ${ }^{3}$ \\ Gilbert A. Esquerdo, ${ }^{3,7}$ Mark E. Everett, ${ }^{7}$ Timothy M. Brown, ${ }^{8,9}$ Markus Rabus, ${ }^{10}$ \\ Juan A. Belmonte, ${ }^{10}$ AND Lynne A. Hillenbrand ${ }^{2}$ \\ Received 2007 June 18; accepted 2007 August 6; published 2007 September 14
}

\begin{abstract}
We report the discovery of TrES-4, a hot Jupiter that transits the star GSC 02620-00648 every 3.55 days. From high-resolution spectroscopy of the star, we estimate a stellar effective temperature of $T_{\text {eff }}=6100 \pm 150 \mathrm{~K}$, and from high-precision $z$ and $B$ photometry of the transit we constrain the ratio of the semimajor axis $a$ and the stellar radius $R_{*}$ to be $a / R_{*}=6.03 \pm 0.13$. We compare these values to model stellar isochrones to constrain the stellar mass to be $M_{*}=1.22 \pm 0.17 M_{\odot}$. Based on this estimate and the photometric time series, we constrain the stellar radius to be $R_{*}=1.738 \pm 0.092 R_{\odot}$ and the planet radius to be $R_{p}=1.674 \pm 0.094 R_{\text {Jup }}$. We model our radial velocity data assuming a circular orbit and find a planetary mass of $0.84 \pm 0.10 M_{\mathrm{Jup}}$. Our radial velocity observations rule out line-bisector variations that would indicate a specious detection resulting from a blend of an eclipsing binary system. TrES-4 has the largest radius and lowest density of any of the known transiting planets. It presents a challenge to current models of the physical structure of hot Jupiters and indicates that the diversity of physical properties among the members of this class of exoplanets has yet to be fully explored.
\end{abstract}

Subject headings: planetary systems — techniques: photometric — techniques: radial velocities techniques: spectroscopic

\section{INTRODUCTION}

Despite the ever increasing number of discovered transiting exoplanets (18 at the time of writing), our understanding of the relationships between host star properties and the planets' physical and orbital parameters is still incomplete. While the massradius relation for most transiting planets agrees with the models (Charbonneau et al. 2007a), several planets have either too high or too low densities. In particular, HD 209458b (Knutson et al. 2007), HAT-P-1b (Bakos et al. 2007), and WASP-1b (Collier Cameron et al. 2007) all have much larger radii and lower densities than expected for planets of their mass and distance from the host star. Several mechanisms for explaining this discrepancy have been proposed, among them different internal heat sources (Guillot \& Showman 2002; Bodenheimer et al. 2003), increased planetary atmospheric opacities (Burrows et al. 2007), and less efficient heat transport as a result of interior composition gradients (Chabrier \& Baraffe 2007). Our Trans-atlantic Exoplanet Survey (TrES) has previously announced the discovery of three transiting planets (Alonso et al. 2004; O'Donovan et al. 2006, 2007), each with distinctive properties. We describe here the newly discovered transiting planet TrES-4, whose mean density of $\rho=0.222 \pm 0.045 \mathrm{~g} \mathrm{~cm}^{-3}$ is the lowest of all known exoplanets with measured radii and masses.

\section{PHOTOMETRY AND SPECTROSCOPY}

We monitored a 5:8 $\times 5.8$ field in Hercules with the Lowell Observatory Planet Search Survey Telescope (PSST; Dunham

\footnotetext{
${ }^{1}$ Lowell Observatory, Flagstaff, AZ; gmand@lowell.edu.

${ }^{2}$ California Institute of Technology, Pasadena, CA.

${ }^{3}$ Harvard-Smithsonian Center for Astrophysics, Cambridge, MA.

${ }^{4}$ Alfred P. Sloan Research Fellow.

${ }^{5}$ Hubble Fellow.

${ }^{6}$ INAF-Osservatorio Astronomico di Torino, Pino Torinese, Italy.

${ }^{7}$ Planetary Science Institute, Tucson, AZ.

${ }^{8}$ Las Cumbres Observatory Global Telescope, Goleta, CA.

${ }^{9}$ University of California, Santa Barbara, CA.

${ }^{10}$ Instituto de Astrofísica de Canarias, La Laguna, Tenerife, Spain.
}

et al. 2004) and the Sleuth telescope at Palomar Observatory between UT 2006 May 6 and 2006 August 2. All images were processed and the photometry and transit search carried out as described in Dunham et al. (2004). Both telescopes detected transits of the host star GSC 02620-00648: PSST observed two full and one partial transits, and Sleuth observed three full and four partial transits. The shape of the events was consistent with the transit of a Jupiter-sized planet across an F dwarf, and we undertook a program of follow-up observations to confirm the planetary nature of the object.

We observed the candidate with the CfA Digital Speedometer (Latham 1992) from 2006 September to 2007 April. We obtained seven spectra covering $45 \AA$ centered at $5187 \AA$, with a resolving power of $\lambda / \Delta \lambda \simeq 35,000$ and signal-to-noise ratio $(\mathrm{S} / \mathrm{N})$ between 11 and 13 per resolution element. This spectral region includes the gravity-sensitive $\mathrm{Mg} b$ triplet, and the host star properties derived from an analysis of this region will depend on the star's metallicity. We obtained the radial velocities (RVs) by crosscorrelation against a synthetic template chosen from a large library of calculated spectra based on Kurucz model atmospheres (see Nordström et al. 1994; Latham et al. 2002). These velocities have a typical precision of $0.5 \mathrm{~km} \mathrm{~s}^{-1}$, and they show no significant variation within the errors. We derived the effective temperature $\left(T_{\text {eff }}\right)$ and projected rotational velocity $(v \sin i)$ by comparing our observed spectra against synthetic spectra with a wide range of parameters (see Torres et al. 2002). The values of $T_{\text {eff }}$ and $v \sin i$ are listed in Table 1 and assume $[\mathrm{Fe} / \mathrm{H}]=0.0$. We also estimate the surface gravity to be $\log g=3.8 \pm 0.2$. We combined the Johnson-Cousins photometry that we obtained (see below) with archive Two Micron All Sky Survey (2MASS) data and used the color-temperature calibrations for dwarfs by Ramírez \& Meléndez (2005) and Casagrande et al. (2006) to estimate $T_{\text {eff }}$. The average result, $T_{\text {eff }}=6130 \pm 80 \mathrm{~K}$, is consistent with the spectroscopic value.

The mass and radius of the star were determined on the basis of the spectroscopic $T_{\text {eff }}$, the value of $a / R_{*}$ derived from the lightcurve fit described below, and an assumed metallicity of 
TABLE 1

TrES-4 Host STAR

\begin{tabular}{|c|c|c|}
\hline Parameter & Value & Referenc \\
\hline R.A. (J2000.0) . & $17^{\mathrm{h}} 53^{\mathrm{m}} 13.05^{\mathrm{s}}$ & 1 \\
\hline Decl. (J2000.0) ...... & $+37^{\circ} 12^{\prime} 42.6^{\prime \prime}$ & 1 \\
\hline GSC & $02620-00648$ & \\
\hline$\left[\mu_{\alpha}, \mu_{\delta}\right] \ldots$ & {$[-6.5,-23.5]$ mas $\mathrm{yr}^{-1}$} & 1 \\
\hline$V \ldots \ldots$ & $11.592 \pm 0.004$ & 2 \\
\hline$B-V$ & $0.520 \pm 0.007$ & 2 \\
\hline$V-\mathrm{R}_{\mathrm{C}}$ & $0.312 \pm 0.008$ & 2 \\
\hline$V-I_{\mathrm{C}} \cdot$. & $0.601 \pm 0.008$ & 2 \\
\hline$J \ldots$ & $10.583 \pm 0.018$ & 3 \\
\hline$J-H \quad \ldots \ldots \ldots \ldots \ldots$ & $0.233 \pm 0.024$ & 3 \\
\hline$J-K_{s}$. & $0.253 \pm 0.026$ & 3 \\
\hline$M_{*} \ldots \ldots \ldots \ldots \ldots \ldots$ & $1.22 \pm 0.17 M_{\odot}$ & 2 \\
\hline$R_{*}{ }^{\mathrm{a}}{ }^{\mathrm{a}} \ldots \ldots \ldots \ldots \ldots \ldots$ & $1.738 \pm 0.092 R_{\odot}$ & 2 \\
\hline$L_{*}, \ldots \ldots \ldots \ldots \ldots \ldots$ & $3.74 \pm 0.86 L_{\odot}$ & 2 \\
\hline$T_{\text {eff }}^{*} \ldots \ldots$ & $6100 \pm 150 \mathrm{~K}$ & 2 \\
\hline $\log g$ & $4.045 \pm 0.034 \mathrm{~cm} \mathrm{~s}^{-2}$ & 2 \\
\hline$v \sin i$ & $9.5 \pm 1.0 \mathrm{~km} \mathrm{~s}^{-1}$ & 2 \\
\hline Age & $4.7 \pm 2.0 \mathrm{Gyr}$ & 2 \\
\hline Distance & $440 \pm 60 \mathrm{pc}$ & 2 \\
\hline
\end{tabular}

${ }^{\text {a }}$ This value of $R_{*}$ is derived from fitting the entire light curve and is better constrained than the value obtained solely from the stellar evolution models $(\S 2)$. The uncertainty in $R_{*}$ includes both the statistical incertainty of $0.044 R_{\odot}$ and the $14 \%$ uncertainty in $M_{*}$.

REFERENCES. - (1) UCAC2 (Zacharias et al. 2004); (2) this Letter; (3) 2MASS (Skrutskie et al. 2006).

$[\mathrm{Fe} / \mathrm{H}]=0.0 \pm 0.2$. The quantity $a / R_{*}$ is closely related to the stellar density and is determined in this case with much higher relative precision than $\log g$. It is therefore a better proxy for luminosity (see Sozzetti et al. 2007). We searched for the best match between stellar evolution models interpolated to a fine grid in age and metallicity and the three observables, within the stated errors. Uncertainties were derived from the full range in $M_{*}$ and $R_{*}$ allowed by the isochrones within observational errors. A comparison with the models from the series by Yi et al. (2001) yielded $M_{*}=1.22 \pm 0.17 M_{\odot}, R_{*}=1.738 \pm 0.092 R_{\odot}$, and $\log g=4.045 \pm 0.034$. This value of $\log g$ is consistent with, but better constrained than, the spectroscopic estimate. The age we derive is $4.7 \pm 2.0 \mathrm{Gyr}$, and the predicted absolute visual magnitude $\left(M_{V}=3.36 \pm 0.27\right)$ implies a distance of $440 \pm$ $60 \mathrm{pc}$, ignoring extinction. The error estimates above do not include possible systematic errors in the stellar evolution models. The gravity, radius, and age estimates for the host star indicate that this is an evolved star at the base of the subgiant branch.

In order to characterize the host star, we obtained off-transit $B V(R I)_{\mathrm{C}}$ photometry of TrES-4 on UT 2007 April 14 with the $1.05 \mathrm{~m}$ Hall telescope at Lowell Observatory in combination with a $2 \mathrm{~K} \times 2 \mathrm{~K}$ SITe CCD. We calibrated the photometry using seven standard fields from Landolt (1992). The results are listed in Table 1 together with other relevant data for the host star of TrES-4.

We carried out high-precision in-transit $z$-band photometry of TrES-4 on UT 2007 May 3 and UT 2007 May 10 with KeplerCam (Holman et al. 2006) at the Fred L. Whipple Observatory $1.2 \mathrm{~m}$ telescope and $B$-band photometry on UT 2007 May 10 using NASACam at the Lowell Observatory $0.8 \mathrm{~m}$ telescope. With KeplerCam, we gathered 373 and $540 z$-band 30 s exposures on UT 2007 May 3 and UT 2007 May 10, respectively. With NASACam, we obtained 19260 s $B$-band exposures. For both data sets, we derived differential fluxes relative to an ensemble of local comparison stars. The photometry is shown in Figure 1.

We obtained high-precision RV measurements of TrES-4 on UT 2007 March 27-29, using the High Resolution Echelle Spec-

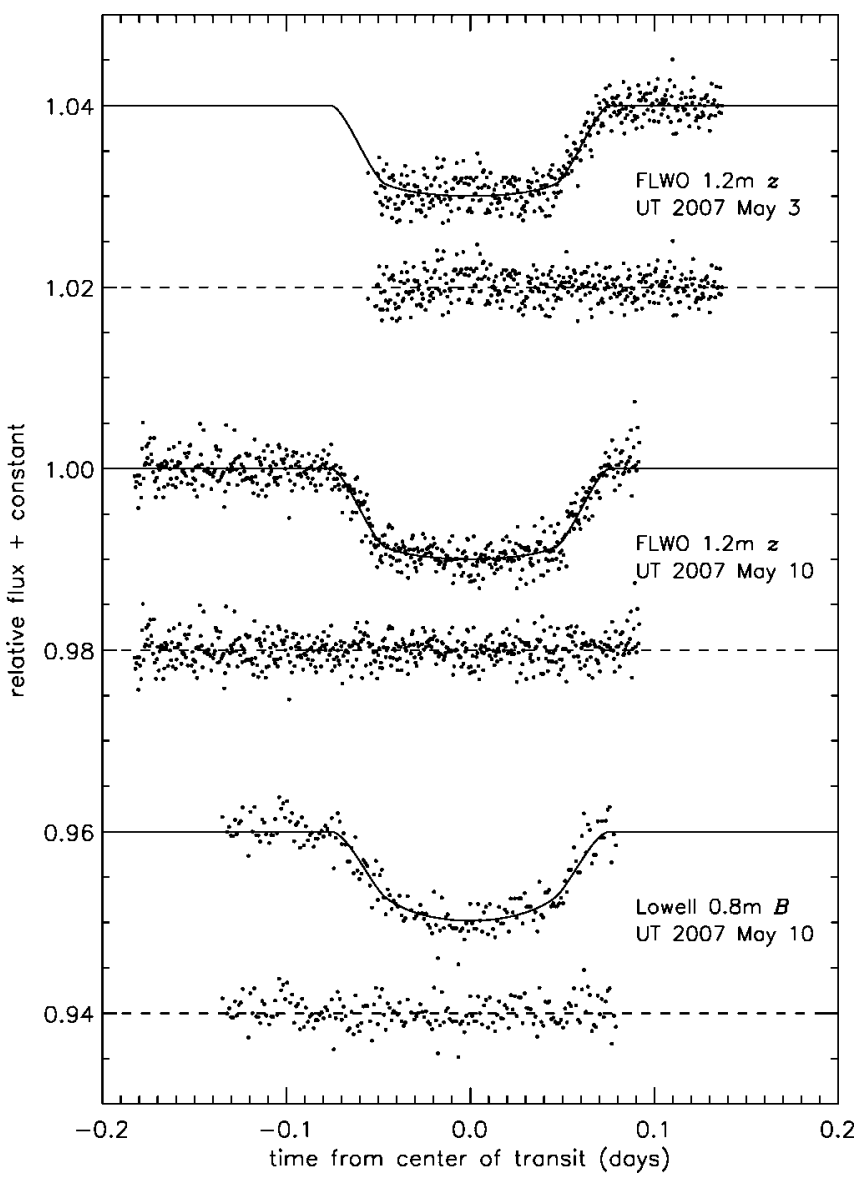

FIG. 1.-High-precision follow-up $z$-band and $B$-band photometry of TrES-4. The plot shows the relative flux (including the color-dependent extinction correction) of the TrES-4 system as a function of time relative to the center of transit, adopting the ephemeris in Table 3 . Each light curve is labeled with the telescope and date of observation. The residuals from the simultaneous fits (solid lines) are shown below each light curve.

trometer (HIRES) and its $\mathrm{I}_{2}$ absorption cell (Vogt et al. 1994) on the Keck I telescope. Our spectra have a nominal resolving power $\lambda / \Delta \lambda \simeq 55,000$ and a typical $\mathrm{S} / \mathrm{N} \sim 120 \mathrm{pixel}^{-1}$. We used nine echelle orders in the wavelength range $3200-8800 \AA$ to derive the velocities for TrES-4. The four star-plus- $\mathrm{I}_{2}$ spectra (plus one $\mathrm{I}_{2}$-free template exposure) provide good coverage of the critical phases. As the ephemeris of the system is fixed, RV data are needed only to determine the amplitude and systemic velocity of the orbit. In these cases (see Konacki et al. 2003), a few measurements are enough. We extracted and reduced all raw spectra using the MAKEE software written by T. Barlow (see Sozzetti et al. 2006). The final RV values are listed in Table 2. The typical precision of the RVs $\left\langle\sigma_{\mathrm{RV}}\right\rangle \simeq 10 \mathrm{~m} \mathrm{~s}^{-1}$ is slightly degraded by the modest stellar rotation. The high-resolution, high-S/N Keck spectra were then used to rule out blend scenarios (see below), and they are presently being analyzed for improved characterization of the host star.

We fit a Keplerian orbit to these data assuming zero eccen-

TABLE 2

Radial Velocity Measurements of TrES-4

\begin{tabular}{ccc}
\hline \hline HJD & & $\begin{array}{c}\text { Radial Velocity } \\
\left(\mathrm{m} \mathrm{s}^{-1}\right)\end{array}$ \\
\hline $2,454,187.07793$ & $\ldots \ldots$ & $97.5 \pm 11.8$ \\
$2,454,188.11122$ & $\ldots \ldots$ & $58.9 \pm 9.4$ \\
$2,454,189.02631$ & $\ldots \ldots$ & $-76.5 \pm 11.6$ \\
$2,454,189.14920 \ldots \ldots$ & $-73.9 \pm 10.3$ \\
\hline
\end{tabular}


TABLE 3

TrES-4 Planet Parameters

\begin{tabular}{lc}
\hline \hline \multicolumn{1}{c}{ Parameter } & Value \\
\hline$P \ldots \ldots \ldots \ldots \ldots \ldots \ldots$ & $3.553945 \pm 0.000075$ days \\
$T_{c} \ldots \ldots \ldots \ldots \ldots \ldots \ldots$ & HJD $2,454,230.9053 \pm 0.0005$ \\
$a \ldots \ldots \ldots \ldots \ldots \ldots \ldots$ & $0.0488 \pm 0.0022 \mathrm{AU}$ \\
$i \ldots \ldots \ldots \ldots \ldots \ldots \ldots$ & $82.81^{\circ} \pm 0.33^{\circ}$ \\
$a / R_{*} \ldots \ldots \ldots \ldots \ldots \ldots$ & $6.026 \pm 0.131$ \\
$b=a \cos i / R_{*} \ldots \ldots$ & $0.755 \pm 0.015$ \\
$K \ldots \ldots \ldots \ldots \ldots \ldots \ldots$ & $97.4 \pm 7.2 \mathrm{~m} \mathrm{~s}^{-1}$ \\
$\gamma_{\text {HIRES }} \ldots \ldots \ldots \ldots \ldots$ & $23.7 \pm 5.8 \mathrm{~m} \mathrm{~s}^{-1}$ \\
$M_{p} \ldots \ldots \ldots \ldots \ldots \ldots$ & $0.84 \pm 0.10 M_{\text {Jup }}$ \\
$R_{p}{ }^{a} \ldots \ldots \ldots \ldots \ldots \ldots \ldots$ & $1.674 \pm 0.094 R_{\text {Jup }}$ \\
$\bar{\rho} \ldots \ldots \ldots \ldots \ldots \ldots \ldots$ & $0.222 \pm 0.045 \mathrm{~g} \mathrm{~cm}^{-3}$ \\
$\log g \ldots \ldots \ldots \ldots \ldots \ldots$ & $2.871 \pm 0.038 \mathrm{~cm} \mathrm{~s}^{-2}$ \\
$R_{p} / R_{*} \ldots \ldots \ldots \ldots \ldots$ & $0.09903 \pm 0.00088$ \\
\hline
\end{tabular}

${ }^{\text {a }}$ The uncertainty in $R_{p}$ includes both the statistical incertainty of $0.053 R_{\mathrm{Jup}}$ and the $14 \%$ uncertainty in $M_{*}$ from Table 1 .

tricity as a good first approximation, as expected from theoretical arguments for a period as short as 3.55 days. The period and epoch of transit were held fixed. The rms of this fit is $11 \mathrm{~m} \mathrm{~s}^{-1}$, which is similar to the internal errors of the velocities. The parameters of this orbital solution are listed in Table 3. We find $M_{p} \sin i=0.731 \pm 0.057\left[\left(M_{*}+M_{p}\right) / M_{\odot}\right]^{2 / 3} M_{\text {Jup }}$. The orbit is displayed in Figure 2 (top panel) along with the observations, and residuals are shown in the middle panel.

We investigated the possibility that the RV variations are the result of distortions in the line profiles caused by contamination from an unresolved eclipsing binary (Santos et al. 2002; Torres et al. 2005), instead of being caused by a planetary companion. We cross-correlated each Keck spectrum against a synthetic template matching the properties of the star and averaged the correlation functions over all orders blueward of the region affected by the $\mathrm{I}_{2}$ lines. From this representation of the average spectral line profile, we computed the mean bisectors, and as a measure of the line asymmetry we calculated the "bisector spans" as the velocity difference between points selected near the top and bottom of the mean bisectors (Torres et al. 2005). If the RV variations were the result of a blend with an eclipsing binary, we would expect the line bisectors to vary in phase with the photometric period with an amplitude similar to that of the velocities (Queloz et al. 2001; Mandushev et al. 2005). Instead, we detect no variation in excess of the measurement uncertainties (see Fig. 2, bottom panel), and we conclude that the RV variations are real and that the star is orbited by a Jovian planet.

\section{PROPERTIES OF TrES-4 AND DISCUSSION}

We analyzed the two $z$-band and one $B$-band photometric time series using the analytic light curves of Mandel \& Agol (2002). We assumed a circular orbit and a quadratic stellar limbdarkening law, fixing the coefficients at the color-dependent values tabulated in Claret $(2000,2004)$ for the spectroscopic $T_{\text {eff }}$ and $\log g$ and assuming solar metallicity. We first estimated the time of center of transit $T_{c}$ by fitting a model light curve to the $z$ data gathered on UT 2007 May 10. We then determined the orbital period $P$ by combining these data with the TrES discovery data (which affords a baseline of $1.0 \mathrm{yr}$ ). We then fixed the values of $T_{c}$ and $P$ (listed in Table 3 ) in the subsequent analysis.

Our model has six free parameters: the planet radius $R_{p}$, the stellar radius $R_{*}$, the orbital inclination $i$, and the color-dependent extinction for each of the three photometric data sets $-k_{z 1}, k_{z 2}$, and $k_{B}$. We assume that the observed flux is proportional to $e^{-k m}$, where $m$ denotes the air mass. The values of $R_{p}$ and $R_{*}$ as

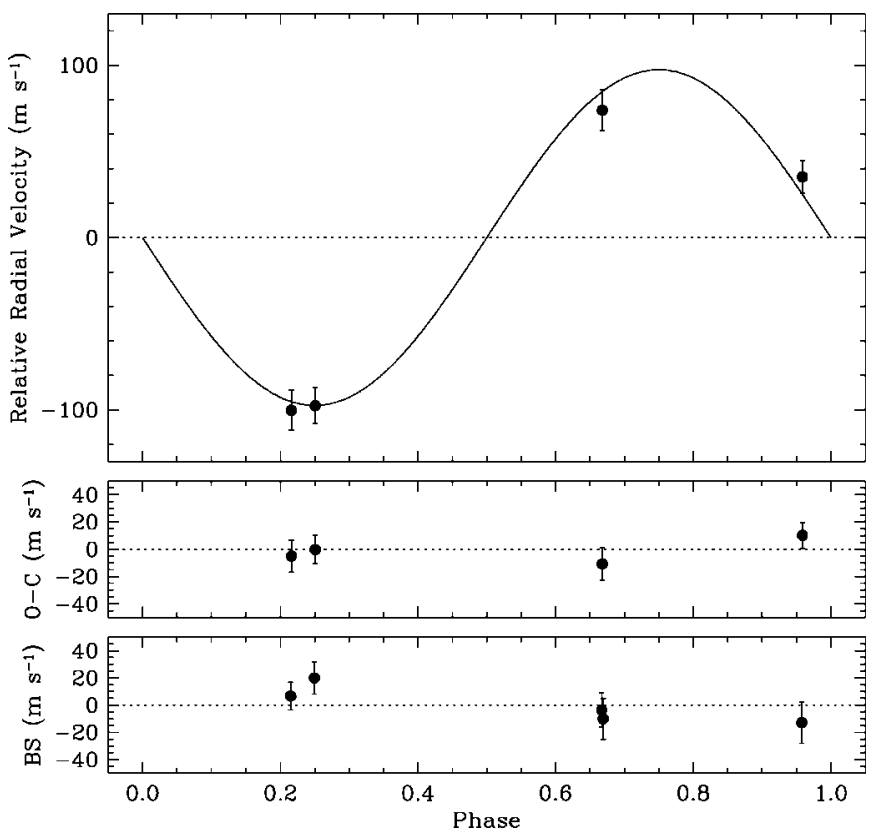

FIG. 2.-Top: Radial velocity observations of TrES-4 obtained with the Keck HIRES using the $\mathrm{I}_{2}$ cell, shown relative to the center of mass and adopting the ephemeris in Table 3. The best-fit orbit (solid line) is overplotted. Middle: Residuals from the best-fit model to the radial velocities. Bottom: Bisector spans shifted to a median of zero, for each of the iodine exposures as well as for the template (which is shown as an additional point at phase 0.669).

constrained by the light curves alone are covariant with $M_{*}$. In our analysis, we first estimated the quantity $a / R_{*}$, which is independent of the assumed value of $M_{*}$, and then used this estimate to constrain the value of $M_{*}$ from stellar isochrones (Sozzetti et al. 2007; Holman et al. 2007). We then fixed $M_{*}=$ $1.22 M_{\odot}$ and estimated the systematic error in the radii using the scaling relations $R_{p} \propto R_{*} \propto M_{*}^{1 / 3}$ (see footnote to Table 1).

We found first the values of $R_{p}, R_{*}, i, k_{z 1}, k_{z 2}$, and $k_{B}$ that minimized the $\chi^{2}$ using the AMOEBA algorithm (Press et al. 1992). This model is shown as the solid curves in Figure 1. We then conducted a Markov Chain Monte Carlo (MCMC) analysis similar to that described in Holman et al. (2006), Charbonneau et al. (2007b), and Winn et al. (2007). We created two MCMC chains with 400,000 points each, one starting from the best-fit values and one starting from a random perturbation to those values. We then rejected the first $23 \%$ of the points to minimize the impact of the initial conditions and found the results from the two chains to be indistinguishable. We examined the histograms of the six parameters as well as the histograms for several combinations of parameters relevant to anticipated follow-up studies. We assigned the optimal value to the median and the $1 \sigma$ errors to the symmetric range about the median that encompassed $68.3 \%$ of the values. We list these estimates in Table 3.

TrES-4 has the largest radius and the lowest density of all exoplanets whose mass and radius are known, and as such presents new challenges for the theory of irradiated gas giants. Burrows et al. (2007) suggested that increased planetary atmospheric opacities and the inclusion of a transit radius correction (Baraffe et al. 2003; Burrows et al. 2003) can explain the radii of all large planets. It appears, however, that TrES-4's radius is still too large for its mass, age, and insolation. In terms of mass and distance to the host star, TrES-4 is similar to HD 209458b but has nearly $30 \%$ larger radius $\left(R_{p}=1.67\right.$ vs. $\left.R_{p}=1.32\right)$. Because of the higher host star luminosity, TrES-4 receives about twice the stellar flux, but it is unlikely that the radius difference can be 
explained solely by the higher stellar irradiation. For example, HAT-P-1b, which has about the same radius as HD 209458b and is the only other planet with $\rho<0.3 \mathrm{~g} \mathrm{~cm}^{-3}$, receives only $60 \%$ of the flux that HD 209458b does. None of the recent models of hot Jupiters (e.g., Fortney et al. 2007; Burrows et al. 2007) can predict a radius as large as that of TrES-4 at its orbital separation for the estimated age and for any mass, even when higher atmospheric opacities are considered and allowance for the transit radius correction is made.

The properties of TrES-4 and its host star allow for many interesting follow-up studies, some of which are already underway. The large radius of TrES-4 in the visual suggests that it may have an extended outer atmosphere, similar to that detected around HD 209458b in Ly $\alpha$ by Vidal-Madjar et al. (2003). If such an extended envelope is caused by mass loss through the planet's Roche lobe boundary, TrES-4 may have an even bigger envelope because of its smaller Roche limit: $R_{\text {Roche }} \approx 3.5 R_{p}$ versus $R_{\text {Roche }} \approx 4.4 R_{p}$ for HD $209458 \mathrm{~b}$ (Erkaev et al. 2007). Moreover, TrES-4 might be in a strong hydrodynamic "blow-off" regime, where the outer atmospheric layers are detached from the planet's gravitational field and escape in a comet-like tail (Vidal-Madjar et al. 2003; Lecavelier des Etangs et al. 2004).
The relatively fast rotation ( $v \sin i=9.5 \mathrm{~km} \mathrm{~s}^{-1}$ ) and brightness of TrES-4's host star, as well as the planet's size, are favorable for measuring the Rossiter-McLaughlin effect (RME) (e.g., Gaudi \& Winn 2007). Of particular interest is the angle between the star's spin axis and the planet's orbital plane, as it can provide information about the possible mechanisms of the planet migration and interaction with the protoplanetary disk. The semiamplitude of the RME is proportional to $v \sin i$ and to $\left(R_{p} / R_{*}\right)^{2}$, and we estimate that for TrES-4 the RME can reach about $90 \mathrm{~m} \mathrm{~s}^{-1}$, roughly equal to the semiamplitude of its orbital velocity.

We thank Travis Barman for useful discussions. This Letter is based on work supported in part by NASA grants NNG04GN74G, NNG04LG89G, NNG05GI57G, NNG05GJ29G, and NNH05AB88I through the Origins of Solar Systems Program, and NASA Planetary Major Equipment grant N4G5-12229. We acknowledge support from the NASA Kepler mission under cooperative agreement NCC2-1390. Work by G. Á. B. was supported by NASA through Hubble Fellowship grant HST-HF-01170.01-A. Observing time on Keck I was awarded by NASA.

\section{REFERENCES}

Alonso, R., et al. 2004, ApJ, 613, L153

Bakos, G. Á., et al. 2007, ApJ, 656, 552

Baraffe, I., et al. 2003, A\&A, 402, 701

Bodenheimer, P., Laughlin, G., \& Lin, D. N. C. 2003, ApJ, 592, 555

Burrows, A., Hubeny, I., Budaj, J., \& Hubbard, W. B. 2007, ApJ, 661, 502

Burrows, A., Sudarsky, D., \& Hubbard, W. B. 2003, ApJ, 594, 545

Casagrande, L., Portinari, L., \& Flynn, C. 2006, MNRAS, 373, 13

Chabrier, G., \& Baraffe, I. 2007, ApJ, 661, L81

Charbonneau, D., Brown, T. M., Burrows, A., \& Laughlin, G. 2007a, in Protostars and Planets V, ed. B. Reipurth, D. Jewitt, \& K. Keil (Tucson: Univ. Arizona Press), 701

Charbonneau, D., et al. 2007b, ApJ, 658, 1322

Claret, A. 2000, A\&A, 363, 1081 2004, A\&A, 428, 1001

Collier Cameron, A., et al. 2007, MNRAS, 375, 951

Dunham, E. W., Mandushev, G., Taylor, B., \& Oetiker, B. 2004, PASP, 116, 1072

Erkaev, N. V., et al. 2007, A\&A, 472, 329

Fortney, J. J., Marley, M. S., \& Barnes, J. W. 2007, ApJ, 659, 1661

Gaudi, B. S., \& Winn, J. N. 2007, ApJ, 655, 550

Guillot, T., \& Showman, A. P. 2002, A\&A, 385, 156

Holman, M. J., et al. 2006, ApJ, 652, 1715

2007, ApJ, 664, 1185

Knutson, H. A., Charbonneau, D., Noyes, R. W., Brown, T. M., \& Gilliland, R. L. 2007, ApJ, 655, 564

Konacki, M., Torres, G., Jha, S., \& Sasselov, D. D. 2003, Nature, 421, 507

Landolt, A. U. 1992, AJ, 104, 340
Latham, D. W. 1992, in ASP Conf. Ser. 32, Complementary Approaches to Double and Multiple Star Research, ed. H. A. McAlister \& W. I. Hartkopeds (San Francisco: ASP), 110

Latham, D. W., et al. 2002, AJ, 124, 1144

Lecavelier des Etangs, A., Vidal-Madjar, A., McConnell, J. C., \& Hébrard, G. 2004, A\&A, 418, L1

Mandel, K., \& Agol, E. 2002, ApJ, 580, L171

Mandushev, G., et al. 2005, ApJ, 621, 1061

Nordström, B., et al. 1994, A\&A, 287, 338

O’Donovan, F. T., et al. 2006, ApJ, 651, L61 2007, ApJ, 663, L37

Press, W. H., Teukolsky, S. A., Vetterling, W. T., \& Flannery, B. P. 1992, Numerical Recipes in C (Cambridge: Cambridge Univ. Press)

Queloz, D., et al. 2001, A\&A, 379, 279

Ramírez, I., \& Meléndez, J. 2005, ApJ, 626, 465

Santos, N. C., et al. 2002, A\&A, 392, 215

Skrutskie, M. F., et al. 2006, AJ, 131, 1163

Sozzetti, A., et al. 2006, ApJ, 649, 428 2007, ApJ, 664, 1190

Torres, G., Konacki, M., Sasselov, D. D., \& Jha, S. 2005, ApJ, 619, 558

Torres, G., Neuhäuser, R., \& Guenther, E. W. 2002, AJ, 123, 1701

Vidal-Madjar, A., et al. 2003, Nature, 422, 143

Vogt, S. S., et al. 1994, Proc. SPIE, 2198, 362

Winn, J. N., Holman, M. J., \& Roussanova, A. 2007, ApJ, 657, 1098

Yi, S. K., et al. 2001, ApJS, 136, 417

Zacharias, N., et al. 2004, AJ, 127, 3043 